\title{
THE
}

\section{Computational Study of the Structures and Thermodynamic Properties of Ammonium Chloride Clusters Using a Parallel Jump- Walking Approach}

\author{
Alexander Matro \\ University of Rhode Island \\ David L. Freeman \\ University of Rhode Island, dfreeman@uri.edu \\ Robert Q. Topper \\ University of Rhode Island
}

Follow this and additional works at: https://digitalcommons.uri.edu/chm_facpubs

Terms of Use

All rights reserved under copyright.

\section{Citation/Publisher Attribution}

Matro, A., Freeman, D. L., \& Topper, R. Q. (1996). Computational Study of the Structures and Thermodynamic Properties of Amonium Chloride Clusters Using a Parallel Jump-Walking Approach. Journal of Chemical Physics, 104(21), 8690-8702. doi: 10.1063/1.471558

Available at: http://dx.doi.org/10.1063/1.471558

This Article is brought to you for free and open access by the Chemistry at DigitalCommons@URI. It has been accepted for inclusion in Chemistry Faculty Publications by an authorized administrator of DigitalCommons@URI. For more information, please contact digitalcommons-group@uri.edu. 


\title{
Computational study of the structures and thermodynamic properties of ammonium chloride clusters using a parallel jump-walking approach
}

\author{
Alexander Matro and David L. Freeman \\ Department of Chemistry, University of Rhode Island, 51 Lower College Road, Kingston, Rhode Island \\ 02881-0809 \\ Robert Q. Topper \\ Department of Chemistry, University of Rhode Island, 51 Lower College Road, Kingston, Rhode Island \\ 02881-0809 and Department of Chemistry, The Cooper Union for the Advancement of Science and Art, Albert \\ Nerken School of Engineering, 51 Astor Place, New York, NY 10003 ${ }^{a}$
}

(Received 17 January 1996; accepted 27 February 1996)

\begin{abstract}
The thermodynamic and structural properties of $\left(\mathrm{NH}_{4} \mathrm{Cl}\right)_{n}$ clusters, $n=3-10$ are studied. Using the method of simulated annealing, the geometries of several isomers for each cluster size are examined. Jump-walking Monte Carlo simulations are then used to compute the constant-volume heat capacity for each cluster size over a wide temperature range. To carry out these simulations a new parallel algorithm is developed using the parallel virtual machine (PVM) software package. Features of the cluster potential energy surfaces, such as energy differences among isomers and rotational barriers of the ammonium ions, are found to play important roles in determining the shape of the heat capacity curves. (C) 1996 American Institute of Physics. [S0021-9606(96)02021-1]
\end{abstract}

\section{INTRODUCTION}

As atomic or molecular aggregates ranging in size from 3 to as many as several thousand monomer units, clusters represent an intermediate state of matter between the finite and the bulk. This intermediate character of clusters has led to considerable interest in their structural and thermodynamic properties. From the perspective of structure, it is frequently possible to determine the potential energy minima, transition states, and the general topography of the potential energy surface. The thermodynamic properties of clusters often mimic bulk materials and have been shown to exhibit distinctive "solid-like" and "liquid-like" features. ${ }^{1-5}$

The examination of the potential energy surfaces of clusters has been a subject of numerous theoretical investigations. A thorough study by Tsai and Jordan has uncovered many of the isomers and transition states of $(\mathrm{Ar})_{n}$, $n=7-13$ clusters described by a Lennard-Jones pair potential. ${ }^{6}$ Studies of $\mathrm{NaCl}$ clusters and cluster ions by Phillips et al. ${ }^{7}$ have shown that clusters bound by ionic forces are capable of forming a rich variety of structural isomers. Complimentary to the study of potential minima is the study of structures and energies of transition states connecting the minima. In addition to the work of Tsai and Jordan, ${ }^{6}$ potential energy surfaces of clusters have been explored for transition states by Davis et al. ${ }^{8}$ and Wales. ${ }^{9}$ The height of the transition state barriers between isomers can play a pivotal role in influencing the isomerization time scale in clusters.

Two approaches have been used to study the thermodynamic properties of clusters. The first approach ${ }^{10,11}$ uses molecular dynamics simulations to identify temperature regions with liquid-like and solid-like behavior. In the solid-like region clusters undergo small-amplitude vibrations about their potential energy minima, while in the liquid-like region

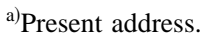

large-amplitude motions are observed, taking the clusters among different potential wells. Lyndon-Bell and Wales ${ }^{12}$ have used molecular dynamics simulations of Lennard-Jones clusters to determine Landau free energy barriers between solid-like and liquid-like states.

A second approach, used in this work, is to use Monte Carlo simulations to calculate thermodynamic properties of the clusters such as $\mathrm{C}_{V}$, the constant-volume heat capacity. Heat capacities for Lennard-Jones clusters ${ }^{1,3}$ have been calculated in this manner, and anomalies in $\mathrm{C}_{V}$ such as peaks and shoulders have been found to correspond to the onset of isomerization. These isomerization transitions have been interpreted as signatures of cluster analogs of phase changes for some cluster sizes. ${ }^{3}$ In addition, Lopez and Freeman ${ }^{13}$ have calculated heat capacities for $\mathrm{Ni}-\mathrm{Pd}$ clusters, observing a "melting" transition and a low-temperature anomaly corresponding to an order-disorder transition analogous to those seen in bulk bimetallic alloys. ${ }^{14}$

Metropolis Monte Carlo simulations ${ }^{15}$ in the temperature region corresponding to the onset of isomerization suffer from the inability to sample all available configuration space ergodically. The deficiency of Metropolis sampling, which cannot be effectively overcome by increasing the step size, is its inability to move among the statistically important regions of configuration space that are separated by significant transition state barriers. One way to overcome the problems of Metropolis sampling is to use the jump-walking (or J-walking) move strategy. ${ }^{1,2} \mathrm{~J}$-walking combines the small step size of Metropolis Monte Carlo with occasional jumps to configurations belonging to a higher temperature ergodic distribution. The higher temperature distribution contains information about the potential energy surface of the system, and the jumps to configurations belonging to separated but statistically important regions of configuration space are thus attempted with sufficient frequency.

Additional complexities in cluster behavior can occur in 
molecular aggregates. For example, in the case of ammonium chloride studied here, the effects from the rotations of the ammonium ions significantly contribute to the thermodynamic properties. The rotational potential energy barriers are, in many instances, smaller than barriers between distinct minima, causing the rotational effects to be reflected in the heat capacity at lower temperatures than the isomerization effects.

In the current work we report two principal outcomes. First, we develop a parallel J-walking algorithm that enables us to carry out a Monte Carlo simulation efficiently in a multi-processor computing environment. Second, the parallel J-walking algorithm is applied to the study of the thermodynamic properties of $\left(\mathrm{NH}_{4} \mathrm{Cl}\right)_{n}, n=3-10$ clusters. The contents of the remainder of this paper are as follows. In Section II we describe the computational methods, including the model potential and a discussion of the simulated annealing methods used to locate the ammonium chloride cluster isomers. This is followed by a discussion of the J-walking Monte Carlo simulation technique used in computing the constant-volume heat capacity, and the implementation of parallel virtual machine (PVM) software ${ }^{16}$ into our Monte Carlo algorithm. Section II concludes with a discussion of the methods used in locating transition states. In Section III we present our results. Isomers of the ammonium chloride clusters found by simulated annealing are shown, along with a few structures of transition states. Then, a constant-volume heat capacity curve for each cluster size is presented, along with a discussion of the features of each curve. Finally, Section IV contains our concluding remarks and directions for future work.

\section{METHODS}

\section{A. Model potential}

In the Monte Carlo simulations carried out in this work, a pair potential dominated by Coulombic interactions is used. The form of the potential is

$V(\mathbf{r})=\sum_{i} \sum_{j>i}\left(A_{i j} \exp \left(-\alpha_{i j} r_{i j}\right)+\frac{q_{i} q_{j}}{r_{i j}}+\frac{D_{i j}}{r_{i j}^{12}}-\frac{C_{i j}}{r_{i j}^{6}}\right)$,

where $\mathbf{r}$ represents the entire set of coordinates for the system, $r_{i j}$ is the distance between particles $i$ and $j$, $q_{\mathrm{Cl}}=-1.0, q_{\mathrm{N}}=-0.4, q_{\mathrm{H}}=0.35$, and the remaining parameters are given in Table I. The potential is derived from potentials of Klein et al. ${ }^{17}$ and Pettitt and Rossky. ${ }^{18,19}$ The parameters not available from other sources are obtained with the standard combination rules. Because the present simulations are classical and the internal vibrations of the ammonium ions are expected to be quantum mechanical with high frequencies, the ammonium ions in the potential of Eq. (1) are assumed to be rigid tetrahedra. With the potential in Eq. (1), in the $\mathrm{CsCl}$ phase at $0 \mathrm{~K}$ the lattice constant for bulk ammonium chloride is found to be $3.79 \AA$ with a cohesive energy of $-720 \mathrm{~kJ} \mathrm{~mol}^{-1}$. These numbers can be compared with the experimental lattice constant $(3.868 \AA)^{20}$ and the experimental cohesive energy at $298 \mathrm{~K}\left(-697 \mathrm{~kJ} \mathrm{~mol}^{-1}\right){ }^{21}$
TABLE I. The parameters used in the model potential. ${ }^{\mathrm{a}}$

\begin{tabular}{lclcc}
\hline \hline Pair & $A_{i j}$ & \multicolumn{1}{c}{$\alpha_{i j}$} & \multicolumn{1}{c}{$C_{6}$} & $D_{12}$ \\
\hline $\mathrm{H}-\mathrm{H}^{\mathrm{b}, \mathrm{c}}$ & 1.0162 & 1.9950 & 2.9973 & 2021.01 \\
$\mathrm{~N}-\mathrm{N}^{\mathrm{d}}$ & 104.74 & 1.5611 & 25.393 & 0 \\
${\mathrm{Cl}-\mathrm{Cl}^{\mathrm{d}}}_{\mathrm{H}-\mathrm{N}^{\mathrm{e}}}^{125.55}$ & 1.7489 & 113.68 & 0 \\
$\mathrm{H}-\mathrm{Cl}^{\mathrm{b}}$ & 10.318 & 1.7780 & 8.7229 & 0 \\
$\mathrm{~N}-\mathrm{Cl}^{\mathrm{e}}$ & 0 & 0 & 10.033 & 43884.0 \\
\hline \hline
\end{tabular}

${ }^{a}$ Units of energy in Hartree and units of length in Bohr.

${ }^{\mathrm{b}}$ Reference 18 .

${ }^{\mathrm{c}}$ Reference 19.

${ }^{\mathrm{d}}$ Reference 17.

${ }^{\mathrm{e}}$ Combining rules.

Because clusters have finite vapor pressures in constant temperature simulations, an external constraining potential ${ }^{22}$ has been included about the center of mass of each cluster. For this constraining potential we have chosen the same form used elsewhere ${ }^{23}$

$$
V_{c}(\mathbf{r})=\kappa \sum_{i=1}^{n}\left(\frac{\left|\mathbf{r}_{i}-\mathbf{R}_{\mathrm{cm}}\right|}{R_{c}}\right)^{20}
$$

where $\mathbf{r}_{i}$ is the coordinate of particle $i, \mathbf{R}_{\mathrm{cm}}$ is the coordinate of the center of mass of the cluster, $\kappa$ has units of energy and $R_{c}$ is a parameter that defines the radius of the constraining potential. In the current calculations $R_{c}$ is taken to be 15 Bohr for the trimer, $20 \mathrm{Bohr}$ for the tetramer, $25 \mathrm{Bohr}$ for $n=5-7$, and 30 Bohr for $n=8-10$. In this work $\kappa$ is taken to be unity.

In the simulated annealing calculations that are used in finding structures of the isomers, it is computationally more convenient to include all degrees of freedom of the system. The internal vibrations of the ammonium ions are described with a harmonic force-field potential of the form

$$
V_{I}=\frac{1}{2} k_{\mathrm{NH}} \sum_{i=2}^{5}\left(r_{1 j}-r_{0}\right)^{2}+\frac{1}{2} k_{\theta} \sum_{i=2}^{4} \sum_{j=i+1}^{5}\left(\theta_{i j}-\theta_{0}\right)^{2},
$$

where atom 1 is the nitrogen, and atoms $2-5$ are the hydrogens, $\theta_{i j}$ is the angle formed by hydrogen atoms $i$ and $j$ and the nitrogen atom, $\theta_{0}$ is the $\mathrm{H}-\mathrm{N}-\mathrm{H}$ angle in a perfect tetrahedron, and $r_{0}=1.9467$ Bohr. The force constants, $k_{\mathrm{NH}}=0.351 \mathrm{Hartree} /(\mathrm{Bohr})^{2}$ and $k_{\theta}=0.137$ Hartree, are given by Herzberg. ${ }^{24}$ The harmonic force-field potential for $\mathrm{NH}_{4}^{+}$ yields vibrational frequencies of 1493, 1692, 3045, and 3193 $\mathrm{cm}^{-1}$. These values compare favorably with the experimental frequencies of $1398,1699,3047$, and $3129 \mathrm{~cm}^{-1}{ }^{25}$

\section{B. Simulated annealing}

The search for the isomers of the $\mathrm{NH}_{4} \mathrm{Cl}$ clusters has been carried out using the method of simulated annealing. This method has been used previously to locate the isomers of other systems. The basic idea of simulated annealing is to take a system at high temperature and gradually cool the system until the global minimum or a local minimum on the potential energy surface is attained. If the cooling is per- 
formed adiabatically, the system will find the global minimum. Using a finite cooling rate allows the system to become trapped in local minima, yielding information about the various isomers.

In this work we use a Brownian dynamics approach. ${ }^{13,26,27}$ Starting with chloride and ammonium ions randomly distributed in a fixed volume, the system is propagated in time according to the Langevin equation. As the temperature is decreased, the kinetic energy is drained from the system. When the temperature finally reaches $0 \mathrm{~K}$, the friction term in the Langevin equation drains the remaining kinetic energy from the system, leaving it in some local minimum.

For the smaller clusters the number of isomers is manageable, and the lowest energy isomer for each cluster size can be identified from the Brownian dynamics simulations. For the larger clusters, however, the number of isomers becomes overwhelming, making it increasingly difficult to identify the lowest energy isomer. We use the J-walking Monte Carlo simulations described below to confirm that the lowest energy isomer is correctly identified. Because the J-walking Monte Carlo simulations are expected to be fully ergodic, the cluster will be in its lowest energy isomer as the simulation temperature approaches $0 \mathrm{~K}$.

Unlike the Monte Carlo simulations, we do not assume rigid ammonium ions in the Brownian dynamics simulations. In most cases the differences between the structures determined by Brownian dynamics and the corresponding rigidammonium structures are small. The main differences consist of slight distortions of the ammonium ions away from tetrahedral geometries and slight distortions of the entire cluster. To find the rigid-ammonium structures from their fully relaxed counterparts, we take the fully relaxed structure and carry out an additional Brownian dynamics simulation at $0 \mathrm{~K}$. During this simulation, force constants $k_{\mathrm{NH}}$ and $k_{\theta}$ in Eq. (3) are gradually increased until the $\mathrm{N}-\mathrm{H}$ bond distances and $\mathrm{H}-\mathrm{N}-\mathrm{H}$ angles are almost at their equilibrium values. Next, the ammonium ions are replaced with perfect tetrahedra, and the energy is further minimized with Monte Carlo moves. All isomers that we have found for $\left(\mathrm{NH}_{4} \mathrm{Cl}\right)_{3}$ and $\left(\mathrm{NH}_{4} \mathrm{Cl}\right)_{4}$, along with the lowest energy isomers for the remaining cluster sizes are presented in Section III.

\section{J-Walking}

It has been seen in several systems that if isomers are separated by high potential energy barriers, Monte Carlo simulations using the ordinary Metropolis move strategy ${ }^{15}$ can result in non-ergodic sampling of configuration space, leading to large errors in calculated averages. This problem can be most acute in the intermediate temperature range at the onset of isomerization. Calculated quantities that suffer most severely from the non-ergodicity of Metropolis Monte Carlo are the quantities involving energy fluctuations, such as the constant-volume heat capacity, $\mathrm{C}_{V}$, given by

$$
\frac{C_{V}}{k_{B}}=\frac{3}{2} n+\frac{\left\langle V^{2}\right\rangle-\langle V\rangle^{2}}{\left(k_{B} T\right)^{2}},
$$

where $\left\langle V^{2}\right\rangle$ and $\langle V\rangle^{2}$ are the average of the square and the square of the average of the potential energy, respectively, $T$ is the temperature, $n$ is the number of particles, and $k_{B}$ is the Boltzmann constant.

The non-ergodicity of the random walk can be alleviated using a move strategy called J-walking. J-walking combines ordinary Monte Carlo moves with jump attempts to configurations in an ergodic distribution at a higher temperature. We discuss briefly $\mathrm{J}$-walking here and refer the reader to original papers for detailed descriptions. ${ }^{1,2}$

For clusters, ordinary Metropolis sampling is normally adequate only for high and low temperatures. At high temperatures, the system is in a fluid-like state, and the combination of high temperature and large Monte Carlo step size is sufficient to overcome barriers among the potential energy wells. On the other hand, only the global minimum is thermally accessible at low temperatures and Metropolis sampling about the global minimum is sufficient. The intermediate temperature range presents a problem for Metropolis sampling because the barriers separating the cluster isomers can result in transitions between potential minima with insufficient frequency.

The main idea behind J-walking is to use information about the potential surface obtained in a high temperature simulation. A walker at a lower temperature executes an ordinary Monte Carlo walk, occasionally attempting jumps to configurations at the higher temperature where the walk is ergodic. Each distinct isomer is sampled adequately with the ordinary Metropolis move strategy, and the occasional jumps to the high temperature distribution ensure that all isomers are included with the proper frequency. Details can be found in the original literature. ${ }^{1,2}$

In practice, J-walking can be implemented in two ways, each with its own set of drawbacks. The first approach uses tandem walkers, one at a high temperature where Metropolis sampling is ergodic, and one or multiple walkers at lower temperatures. The configurations generated by the high temperature walker are used by the lower temperature walkers for attempting J-walking moves. This tandem approach has been used infrequently because correlations inherent in the Metropolis walks can introduce systematic errors in the J-walking results.

The second approach writes the configurations from the simulation at the J-walking temperature to an external file and accesses configurations randomly from the external file while carrying out a simulation at the lower temperature. Correlation errors are avoided with external configuration files because of two features in this approach. First, as discussed in Ref. 3, by writing configurations to an external file infrequently (about once every 40-100 moves), the correlations are partially broken. Second, because correlations still persist between configurations separated by 40-100 moves, it is also necessary to access the external files randomly. The difficulty with this approach is the storage requirements for the external distributions. The large storage requirements have limited the application of the method only to small systems. 


\section{Parallel strategy}

As we discussed above, J-walking can be implemented using either tandem walkers or previously generated external distributions. The best features of these two approaches can be combined into a single J-walking algorithm with the use of multiple processors and the parallel virtual machine (PVM) software package. ${ }^{16}$ PVM enables processes running on the same or different machines to exchange information while they are executing. We incorporate the PVM subroutines into the Monte Carlo computer code, where these subroutines are used to send and receive configuration geometries and potential energies of the clusters.

Using a multiple-processor computing environment, we can execute a separate Monte Carlo walk on each processor, with PVM enabling these Monte Carlo walks to communicate with each other. Instead of generating external distributions and storing them before the actual simulation, we generate the required distributions " on the fly" and pass them to the lower-temperature walkers. The walker accepts or rejects the configuration it receives based on Eq. (5) of Ref. 1.

Our computational scheme consists of two kinds of Monte Carlo processes, the configuration generating processes and the computing processes. The generating processes are designed to be a source of ergodic distributions at particular temperatures. Each set of computing processes carries out Monte Carlo walks at temperatures below that of the generating process, periodically jumping to a configuration provided by the generating process to maintain ergodicity.

A diagram of a model PVM J-walking simulation is shown in Fig. 1. Each box represents a process in a parallel machine executing a Metropolis simulation at a particular temperature. The set of boxes on the left-hand side of Fig. 1 represents the generating processes, executing walks at temperatures $T_{1}, T_{2}$, and $T_{3} . T_{1}$ is assumed to be sufficiently high that Metropolis Monte Carlo is ergodic without modification. The set of boxes on the right-hand side of Fig. 1 represent the computing processes. The necessity of having the generating processes running at different generating temperatures is precisely analogous to generating external distributions at these temperatures in a J-walking simulation using the serial algorithm. ${ }^{1}$ As in the serial J-walking method, a new distribution is needed when the jump acceptance rate falls below $10 \%-15 \%$. The generating processes at $\mathrm{T}_{2}$ maintain their ergodicity just like the computing processes by making jump attempts to configurations contained in the $T_{1}$ processes. In turn, the $T_{2}$ processes serve as sources of ergodic distributions for computing processes executing at temperatures between $T_{2}$ and $T_{3}$. Additionally the generating processes at $T_{3}$ are made ergodic by periodic jumps to the configurations contained in the $\mathrm{T}_{2}$ processes. If still lower temperature generating processes are needed, this procedure can be continued. It is important to note that four processes are executed at each generating temperature. The four downward arrows from $T_{1}$ to $T_{2}$ and from $T_{2}$ to $T_{3}$ denote that the generating processes at each temperature are independent of each other. The boxes on the right-hand side of Fig. 1 represent the computing processes. The thick ar-

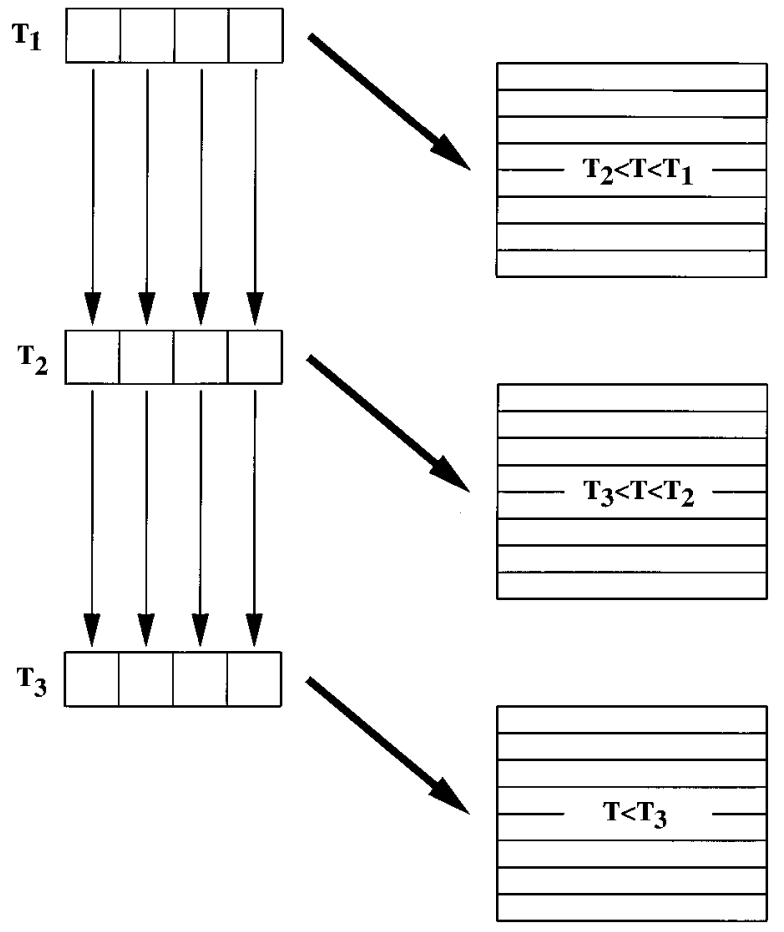

FIG. 1. Diagram of a sample PVM J-walking process. Boxes on the lefthand side represent the generating processes, boxes on the right-hand side represent the computing processes, and arrows indicate the direction in which configurations are passed from the generating processes.

rows pointing from the generating processes to the computing processes indicates that each of the four generating processes is feeding configurations to each computing process.

The reason for having four generating processes at each temperature instead of just one is the following. The bottleneck of this algorithm is the frequency at which a generating process can pass a configuration to a computing process or to a lower-temperature generating process. This frequency must be kept sufficiently low to allow the Monte Carlo simulation at the generating temperature ample opportunity to alter the configuration of the system. Previous J-walking studies of Lennard-Jones clusters have shown that a configuration can be saved once every 40-100 Monte Carlo passes, depending on the size of the cluster. ${ }^{3}$ It has also been determined that a computing process should attempt jumps to the ergodic distribution as often as once every $10-15$ passes. We can maintain the $10-15$ pass criterion in the computing process by having each computing process access four independent generating processes. The result is that each generating process is accessed once every 60 moves, but since there are four, the computing process can attempt a jump to one of the four generating processes once every 15 moves. In tests performed on $\left(\mathrm{NH}_{4} \mathrm{Cl}\right)_{9}$, storing a configuration every 100 passes instead of every 60 did not affect the calculated heat capacity. The computing time is reduced by a factor of 4 in the case where the number of processors is not limited.

As in the serial algorithm there are correlations in the Metropolis walks of each generating process that must be broken. To break the correlations, each configuration- 
generating process keeps an array of configurations in memory. When a configuration is transmitted to a lowertemperature process, it is a configuration randomly chosen from this array, not the current configuration of the walker. The current configuration of the walker then replaces the configuration just passed from the array to another processor. The minimum size for this array has been found to be 2500 configurations from tests on the $\left(\mathrm{NH}_{4} \mathrm{Cl}\right)_{9}$ cluster. In all calculations presented below, a 5000-configuration array is used. Compared to array sizes of at least 50000 configurations used in the serial algorithm, the arrays in the parallel method are small and do not inhibit applications of the method to large systems.

An issue that must be addressed is the initiation of the 5000-configuration array at each generating temperature $\mathrm{T}_{1}, \mathrm{~T}_{2}$, etc. We have examined two approaches. One approach is to create the arrays during the computation. The startup time required to populate the configuration arrays sequentially can be prohibitively long. For example, the computing processes between $T_{1}$ and $T_{2}$ and the generating processes at $T_{2}$ must wait until the 5000-configuration arrays at $\mathrm{T}_{1}$ are created. The other approach, which we have found to be more useful, is to create the small 5000-configuration distributions using serial $\mathrm{J}$-walking prior to the PVM calculation. The initiating walks are short and require storage of arrays of only 5000 configurations. This approach allows the most efficient use of the multi-processor computing environment because there are no idle processors at any time during the PVM calculation.

For the computations in the present work the typical number of Monte Carlo passes for each temperature point is 1.5 million for cluster sizes 4-10. During each run, $10^{5}$ J-walking attempts are made. The calculations for the $\left(\mathrm{NH}_{4} \mathrm{Cl}\right)_{3}$ cluster consist of 3 million moves for each run and $5 \times 10^{4} \mathrm{~J}$-walking attempts. A typical PVM J-walking simulation for the larger ammonium chloride clusters requires a total of about 90 processes to span the entire temperature range of interest. For these calculations, 45 individual processors are used, with two processes executing on each processor.

\section{E. Locating transition states}

Although the energies and geometries of local minima on the multi-dimensional potential energy surface of a cluster are important determining factors for the onset of isomerization, a crucial role is also played by the height of the potential energy barriers separating the minima. Determining the height of these barriers entails the search for the transition states on the potential energy surface.

We use two methods to search for transition states. The first method is an approach previously developed to find the transition states of Lennard-Jones clusters. ${ }^{28}$ Briefly, transition states are located by constructing double-ended classical trajectories that connect a pair of local minima. The trajectories are located by expressing the path between two isomers of interest with a Fourier expansion about a constantvelocity path. The Fourier coefficients are then determined, yielding a trajectory with a particular total energy. The details of finding the Fourier coefficients are given elsewhere. ${ }^{28,29}$ The trajectory with the lowest total energy is usually found to pass through or near the transition state, identified by having exactly one negative eigenvalue in its Hessian matrix.

The other method used in locating the transition states is the eigenmode-following technique initially proposed by Hildenbrand ${ }^{30}$ and developed further by other workers. ${ }^{31}$ More recently, Tsai and Jordan ${ }^{6}$ and Wales et al. ${ }^{9}$ have employed this method to locate transition states of atomic and molecular clusters. Briefly, a search is conducted by starting at a local minimum and following one of the normal modes by maximizing the potential energy along that particular mode while minimizing the potential energy along the remaining normal modes, until a transition state is reached.

For the case of $\left(\mathrm{NH}_{4} \mathrm{Cl}\right)_{4}$ and $\left(\mathrm{NH}_{4} \mathrm{Cl}\right)_{9}$ we are concerned with rotational barriers of the ammonium ions. The double-ended trajectory method for finding transition states is ideally suited to these motions because of their simplicity. For the more complex transition states of the $\left(\mathrm{NH}_{4} \mathrm{Cl}\right)_{3}$ cluster connecting distinct isomers we use the eigenmodefollowing method. The double-ended trajectory method is unable to locate the transition states between the isomers of the trimer with a low number of Fourier expansion coefficients. To apply the double-ended trajectory approach to a case with a complex rearrangement, a large number of Fourier coefficients is required to describe the path of the system between the two minima, making the method computationally expensive. Rather than pursuing the transition state search with the double-ended method, we have opted to use the eigenmode-following method in the transition state searches in $\left(\mathrm{NH}_{4} \mathrm{Cl}\right)_{3}$.

\section{RESULTS}

\section{A. Structures}

The ammonium chloride monomer is known ${ }^{32}$ to be a van der Waals complex rather than an ionic species and cannot be treated using the model potential of Section II A. We do not investigate $\left(\mathrm{NH}_{4} \mathrm{Cl}\right)_{2}$ because it has only a single isomer and is not expected to exhibit interesting thermodynamic behavior. We assume that all clusters in this work are ionic. The cluster size at which there is a transition from van der Waals to ionic bonding represents an interesting and complex electronic structure problem that is beyond the scope of this work.

We begin our discussion of the ammonium chloride cluster structures with $\left(\mathrm{NH}_{4} \mathrm{Cl}\right)_{3}$, for which we have found three isomers with our potential model. These three isomers, located with the simulated annealing procedure described in the previous section, are shown in Figs. 2(a), 2(c), and 2(e). Structures (b) and (d) in Fig. 2 are the transition states connecting (a) with (c) and (a) with (e), respectively. The transition states shown in Figs. 2(b) and 2(d) are determined using the full potential, including the internal vibrations of the ammonium ions. Because our transition state search methods determine the transition states using the full poten- 


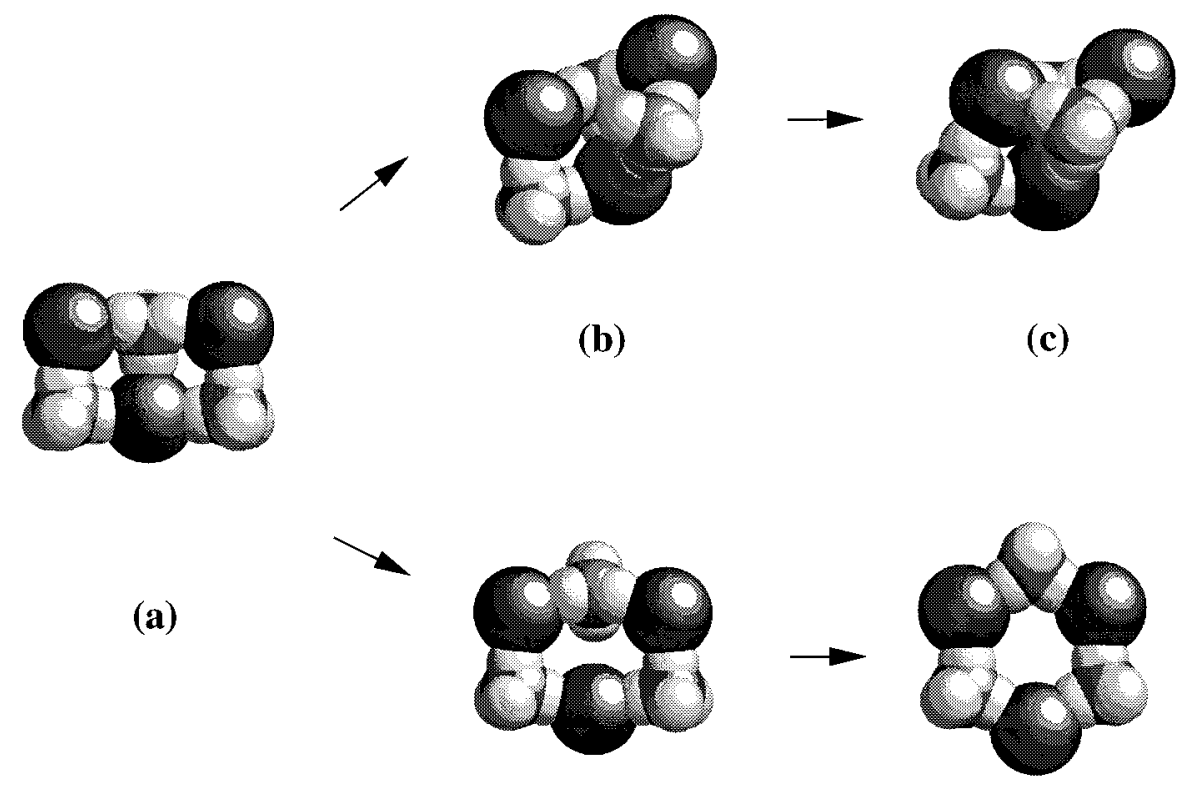

(d)

(e)

FIG. 2. $\left(\mathrm{NH}_{4} \mathrm{Cl}\right)_{3}$ (a) is the lowest energy isomer, $V=-0.69206 \mathrm{eV}$, (c) is the isomer $235 \mathrm{~K}$ above (a), (e) is the isomer $635 \mathrm{~K}$ above (a), (b) is the transition state connecting (a) with (c), $2118 \mathrm{~K}$ above (a), (d) is the transition connecting (a) with (e), $2021 \mathrm{~K}$ above (a). The energies of the minima are determined using Eq. (1). The transition state barriers include intramolecular contributions from Eq. (2).

tial, all transition state barriers reported in this work are calculated relative to the potential energy minima of the full potential. The corresponding transition state barriers for the structures with rigid ammonium ions are expected to be roughly the same. Interestingly, isomers (a) and (c) are so close to each other in energy that isomer (c) becomes the lowest energy isomer if the full potential is used and the ammonium ions are allowed to relax fully.

Figures 3(a)-3(f) show the isomers we located for the $\left(\mathrm{NH}_{4} \mathrm{Cl}\right)_{4}$ cluster in the order of increasing potential energy.

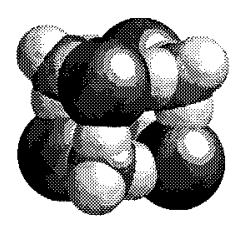

(a)

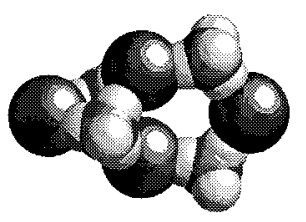

(d)

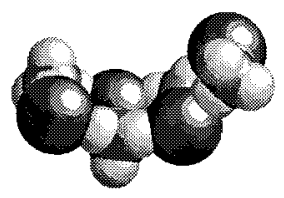

(b)

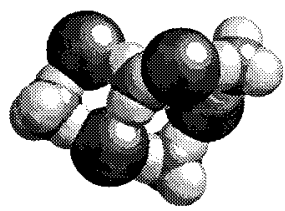

(e)

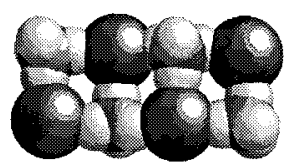

(c)

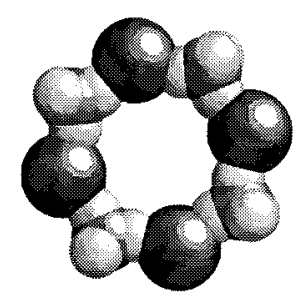

(f)

FIG. 3. All discovered isomers found for $\left(\mathrm{NH}_{4} \mathrm{Cl}\right)_{4}$, (a) is the lowest energy isomer at $V=-0.98950 \mathrm{eV}$, the energies for the remaining isomers are given relative to the lowest energy isomer: (b) $13485 \mathrm{~K}$, (c) $13844 \mathrm{~K}$, (d) $16590 \mathrm{~K}$, (e) $18535 \mathrm{~K}$, (f) $18900 \mathrm{~K}$. 


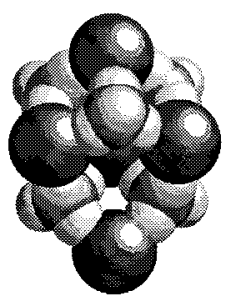

(a)

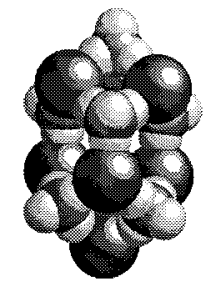

(c)

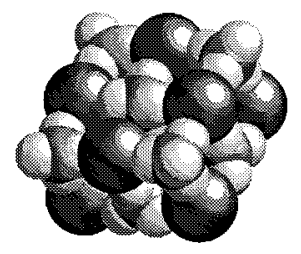

(e)

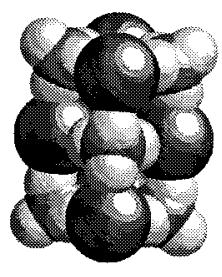

(b)

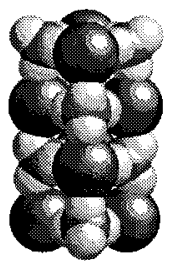

(d)

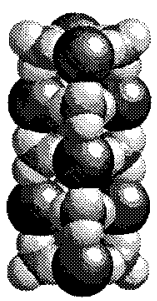

(f)
FIG. 4. The lowest energy isomers for $\left(\mathrm{NH}_{4} \mathrm{Cl}\right)_{n}, n=5-10$; (a) $n$ $=5, V=-1.22880 \mathrm{eV}$, (b) $n=6, V=-1.519387 \mathrm{eV}$, (c) $n=7, V$ $=-1.76779 \mathrm{eV}$, (d) $n=8, V=-2.05161 \mathrm{eV}$, (e) $n=9, V=-2.31868 \mathrm{eV}$, (f) $n=10, V=-2.58333 \mathrm{eV}$.

There is a qualitative difference in structure between the lowest isomer (a) and the remaining isomers (b)-(f). The lowest energy isomer has a compact rock-salt structure while the remaining isomers have open-type structures. Unlike $\left(\mathrm{NH}_{4} \mathrm{Cl}\right)_{3}$, in which all three isomers are close to each other in energy, there is a $13485 \mathrm{~K}$ gap separating the two lowest lying isomers of the tetramer, (a) and (b).

Figure 4 shows the lowest energy isomers for clusters sizes 5-10. It is interesting to note the trend in the growth pattern for the clusters with an even number of ion pairs. Beginning with $\left(\mathrm{NH}_{4} \mathrm{Cl}\right)_{4}$ (see Fig. 3(a)), each successive even-number cluster is formed via the addition of an identical $\left(\mathrm{NH}_{4} \mathrm{Cl}\right)_{2}$ unit. The odd-number clusters do not show a pattern of formation from either the smaller odd-number cluster or the preceding even-number cluster.

The relative stability of the even-number clusters versus the odd-number clusters can be compared by examining the gain in binding energy of the lowest energy isomer, $\Delta V=V_{n-1}-V_{n}$ as a function of the cluster size $n$, where $V_{n}$ is the potential energy of the lowest energy isomer of size, $n$. The trend observed in Fig. 5 clearly shows that the

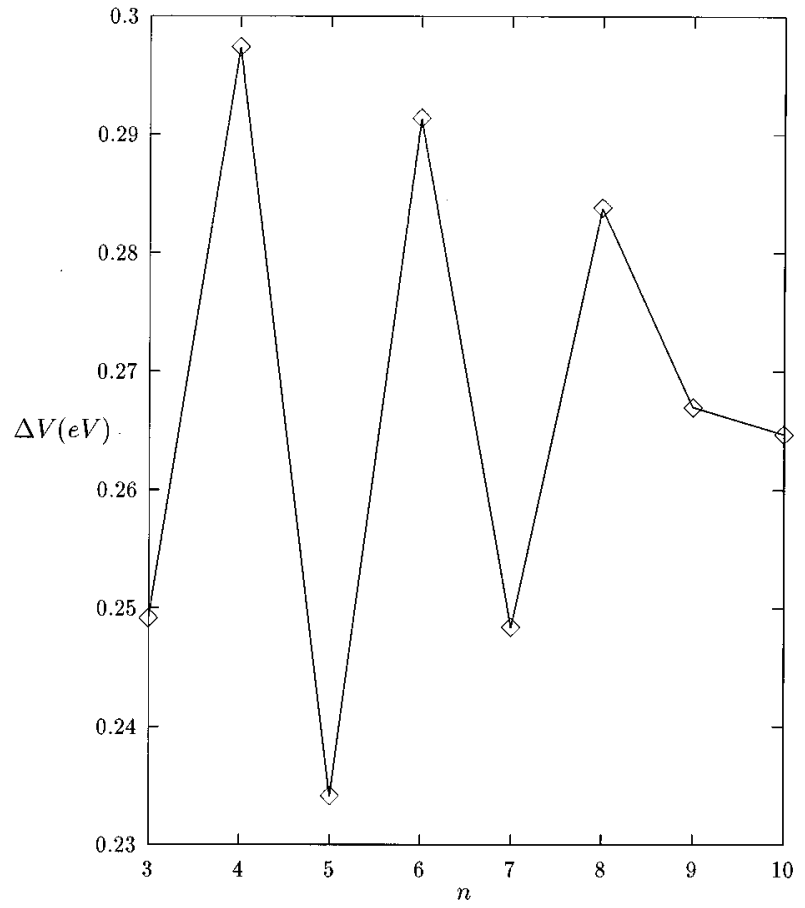

FIG. 5. $\Delta V=V_{n-1}-V_{n}$ as a function of $n$, for the lowest energy isomers. The gain in binding energy for the even clusters is generally greater than the gain for the odd clusters.

gain in potential energy is greater when going from an odd to an even cluster, except for $\left(\mathrm{NH}_{4} \mathrm{Cl}\right)_{9}$. The deviation of $\left(\mathrm{NH}_{4} \mathrm{Cl}\right)_{9}$ from the observed trend can be understood by examining its geometry, shown in Fig. 4(e). The $\left(\mathrm{NH}_{4} \mathrm{Cl}\right)_{9}$ cluster forms a rock-salt structure that has more in common with the even-number clusters than with the other oddnumber clusters.

\section{B. Heat capacities}

The constant-volume heat capacity curve for $\left(\mathrm{NH}_{4} \mathrm{Cl}\right)_{3}$ is shown in Fig. 6. The error bars in this and all subsequent heat capacity curves represent two standard deviations. Also, only the potential energy contribution to the heat capacity is shown in this and all subsequent heat capacity curves, i.e., we set

$$
C_{v}=\frac{\left\langle V^{2}\right\rangle-\langle V\rangle^{2}}{\left(k_{B} T\right)^{2}} .
$$

There are no additional structural features from the constant kinetic energy contribution in Eq. (4). The curve starts at approximately 10.5 , the equipartition value for the trimer, undergoes a slow increase in the $0-80 \mathrm{~K}$ range, then rises rapidly to a peak at $140 \mathrm{~K}$, and finally the heat capacity declines gradually over the remaining temperature range. The presence of an early peak in the heat capacity is a direct consequence of the small differences in energy among the isomers.

We have found that during the simulation at $50 \mathrm{~K}$ all configurations are in the potential energy wells belonging to 


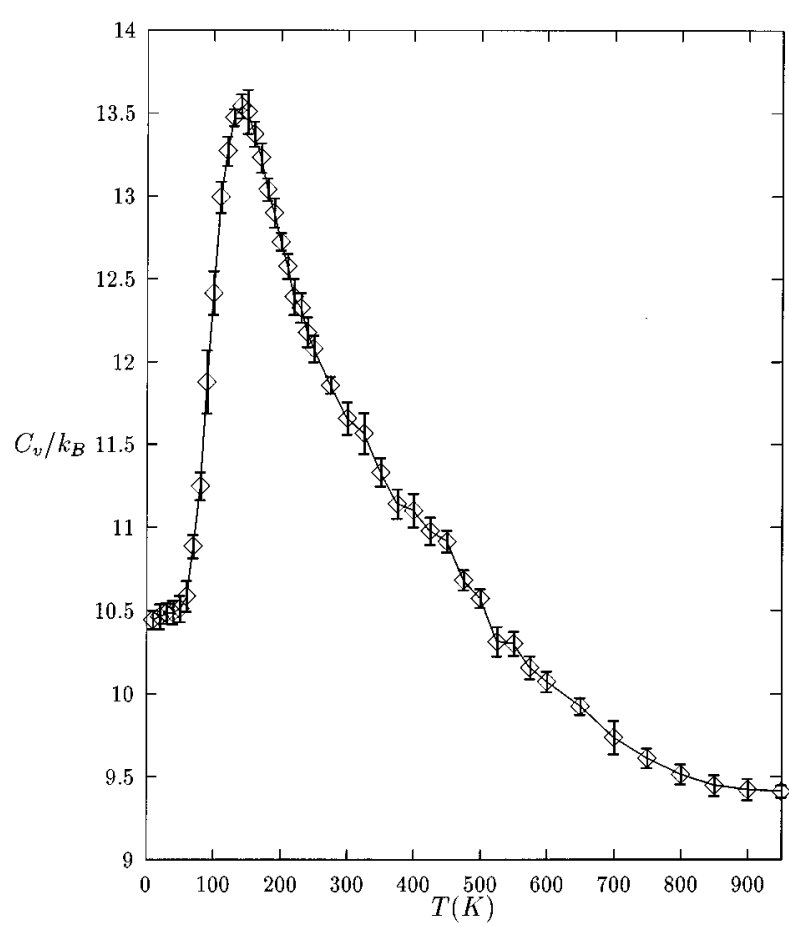

FIG. 6. The potential energy contribution to the constant-volume heat capacity for $\left(\mathrm{NH}_{4} \mathrm{Cl}\right)_{3}$ as a function of $T$.

isomers (a) and (e) in Fig. 2, and none are found in the well belonging to isomer (c). At $250 \mathrm{~K}$, less than $0.03 \%$ of configurations are found in the well of isomer (c). The isomers occupied during a finite-temperature simulation are identified using the nitrogen-chloride distances and the angle between the planes defined by the nitrogens and the chlorides. Since the absence of structures belonging to the potential energy well of isomer (c) is clearly not a consequence of energetics, we have determined transition state barriers between isomers (a) and (c) and between isomers (a) and (e). These transition state geometries are shown in Figs. 2(b) and 2(d), respectively. As discussed in Section III A and listed in Fig. 2, the transition state barriers in both cases are about $2000 \mathrm{~K}$ above isomer (a). The difference in the potential energy barrier heights does not appear to be the reason for the extremely rare occurrence of isomer (c) in our simulations. One possible explanation for the small contribution of structures belonging to the potential well of isomer (c) may be the small fraction of configuration space available to the isomer. A normal mode analysis of isomers (a) and (c) has revealed that the lowest vibrational frequency of isomer (a) is approximately one-half of the the corresponding frequency of isomer (c). Furthermore, the lowest vibrational frequency of isomer (e) is one-third of the corresponding frequency of isomer (c). The frequencies of the first six vibrational modes of isomer (a) are 36, 41, 57, 67, 86, and $94 \mathrm{~cm}^{-1}$. For isomer (c) these are $74,76,107,150,159$, and $163 \mathrm{~cm}^{-1}$, and for isomer (e), the frequencies are 26, 26, 36, 42, 42, and 54 $\mathrm{cm}^{-1}$. Although this difference in normal mode frequencies is not a direct measure of the relative fraction of configura- tion space occupied by the respective isomers, it is nonetheless a reflection of the narrowness of the potential energy well of isomer (c) relative to isomers (a) and (e).

The heat capacity curve for the $\left(\mathrm{NH}_{4} \mathrm{Cl}\right)_{4}$ cluster, shown in Fig. 7, is significantly different from that of $\left(\mathrm{NH}_{4} \mathrm{Cl}\right)_{3}$ in Fig. 6. The peak in the $\left(\mathrm{NH}_{4} \mathrm{Cl}\right)_{4}$ heat capacity occurs at $1100 \mathrm{~K}$. The heat capacity increases slowly until $800 \mathrm{~K}$, at which point it rises rapidly to the aforementioned peak at $1100 \mathrm{~K}$. By examining the structures of $\left(\mathrm{NH}_{4} \mathrm{Cl}\right)_{4}$ during the simulations, we have found that this large peak is a consequence of isomerization transitions to the open structures shown in Fig. 6. For $\left(\mathrm{NH}_{4} \mathrm{Cl}\right)_{4}$ and the remaining clusters we shall define the "melting transition region" to be the range of temperatures where isomerizations to such open structures take place. Another feature of the heat capacity curve shown in Fig. 7 is its almost flat slope in the 400-700 K region. This particular feature is present in most of the cases studied in this paper. As we shall discuss in the context of $\left(\mathrm{NH}_{4} \mathrm{Cl}\right)_{9}$, the temperature region where $C_{v}$ is flat is the region where the $\mathrm{NH}_{4}^{+}$ions are nearly freely rotating. Using the method of double-ended classical trajectories, the barrier for rotation of a $\mathrm{NH}_{4}^{+}$ion about the $\mathrm{N}-\mathrm{H}$ bond pointing outward from the cluster has been determined to be $4300 \mathrm{~K}$.

The heat capacity curve for $\left(\mathrm{NH}_{4} \mathrm{Cl}\right)_{5}$ is shown in Fig. $8(\mathrm{a})$. It has qualitative similarities to the $\left(\mathrm{NH}_{4} \mathrm{Cl}\right)_{4}$ heat capacity shown in Fig. 7 with a melting peak in the 1000-1100 $\mathrm{K}$ region. The melting peak is, however, significantly smaller relative to the $T=0 \mathrm{~K}$ heat capacity than in $\left(\mathrm{NH}_{4} \mathrm{Cl}\right)_{4}$. This is a trend that we observe for all the odd-number clusters, with the exception of $\left(\mathrm{NH}_{4} \mathrm{Cl}\right)_{9}$; namely the ratio of the maximum heat capacity relative to the $T=0 \mathrm{~K}$ value is significantly larger for the even-number clusters than for oddnumber clusters.

Unlike the tetramer, $\left(\mathrm{NH}_{4} \mathrm{Cl}\right)_{5}$ has several low-energy isomers. Isomerization in $\left(\mathrm{NH}_{4} \mathrm{Cl}\right)_{5}$ is seen at significantly lower temperatures than in the tetramer. The isomerization at the lower temperatures in $\left(\mathrm{NH}_{4} \mathrm{Cl}\right)_{5}$ occurs among isomers with compact structures, two of which are shown in Figs. 9(a) and 9(b). Figures 9(c) and 9(d) show examples of higher-energy open structures of $\left(\mathrm{NH}_{4} \mathrm{Cl}\right)_{5}$. The open-type structures are at least $12000 \mathrm{~K}$ above the lowest-energy isomer and become accessible in the vicinity of the 1000-1100 $\mathrm{K}$ melting peak. In contrast, only the lowest energy isomer of $\left(\mathrm{NH}_{4} \mathrm{Cl}\right)_{4}$ has a compact structure and the remaining isomers are all high energy open structures (see Fig. 3).

Figure 8(b) shows the heat capacity curve for $\left(\mathrm{NH}_{4} \mathrm{Cl}\right)_{6}$, and the lowest energy isomer is shown in Fig. 3(b). The heat capacity curve is similar to that of $\left(\mathrm{NH}_{4} \mathrm{Cl}\right)_{4}$. The region between $500 \mathrm{~K}$ and $800 \mathrm{~K}$ is remarkably flat, and the melting peak at $1200 \mathrm{~K}$ is sharp compared with $\left(\mathrm{NH}_{4} \mathrm{Cl}\right)_{5} \cdot\left(\mathrm{NH}_{4} \mathrm{Cl}\right)_{6}$ has at least one other low-energy isomer (not shown). These low-energy isomers of $\left(\mathrm{NH}_{4} \mathrm{Cl}\right)_{6}$ are $2051 \mathrm{~K}$ apart and isomerization between them is seen at temperatures as low as $400 \mathrm{~K}$. The remaining isomers found in this work are open-type structures and are in the range of 12000-14000 K above the lowest energy isomer, becoming accessible at temperatures near the melting peak.

The lowest energy isomer for the $\left(\mathrm{NH}_{4} \mathrm{Cl}\right)_{7}$ cluster is 
shown in Fig. 3(c). The heat capacity curve, shown in Fig. $8(\mathrm{c})$, is the only curve in this study that clearly exhibits two distinct peaks. The first peak occurs around $600 \mathrm{~K}$ and is in the same region where other cluster sizes (most notably 4, 6, 8 , and 9) show a flat region. In this case, however, the peak at $600 \mathrm{~K}$ is a result of isomerization between the global minimum and a local minimum approximately $2000 \mathrm{~K}$ above the global minimum. The second peak, centered at about 1200 $\mathrm{K}$, is a melting peak indicating the onset of isomerization to open-type structures.

To investigate further the origin of the $600 \mathrm{~K}$ peak in the heat capacity of $\left(\mathrm{NH}_{4} \mathrm{Cl}\right)_{7}$, we have performed quench studies of the configurations obtained from the simulations at temperatures of 425,600 , and $800 \mathrm{~K}$. At each temperature, configurations were taken each 750 Monte Carlo passes, and 400 configurations at each temperature were quenched to the nearest local minimum using Brownian dynamics. At $425 \mathrm{~K}$, well before the peak in the heat capacity, almost all 400 configurations from an ergodic distribution quench to the lowest energy isomer. The dominance of the lowest energy isomer is reflected in the large peak in the histogram in Fig. 10 (a) at $0 \mathrm{~K}$ and the small peaks representing higher energy isomers (the energies of the isomers in Fig. 10 are given relative to the lowest energy isomer defined to be $0 \mathrm{~K}$ ). At $600 \mathrm{~K}$ (Fig. 10(b)) slightly more than half of the configurations quench to the lowest energy isomer, with the majority of remaining configurations distributed between two isomers $830 \mathrm{~K}$ and $2194 \mathrm{~K}$ above the lowest energy isomer. The time-scale for isomerization at $600 \mathrm{~K}$ is still slow, corroborated by examining configurations of the $\left(\mathrm{NH}_{4} \mathrm{Cl}\right)_{7}$ cluster saved during the simulation. The infrequent isomerization events result in large fluctuations in the potential energy, leading to a peak in the heat capacity at $600 \mathrm{~K}$. Finally, at $800 \mathrm{~K}$ (see Fig. 10(c)) two isomers are seen to dominate the histogram with roughly equal populations. Rapid isomerization near $800 \mathrm{~K}$ leads to the observed decrease in the heat capacity. The second peak at $1200 \mathrm{~K}$ is the melting peak.

To determine the features of $\left(\mathrm{NH}_{4} \mathrm{Cl}\right)_{7}$ that are unique in this series, we compare the energetics of the clusters. $\left(\mathrm{NH}_{4} \mathrm{Cl}\right)_{4}$ has only one compact structure, the global minimum (see Fig. 3), and the rest of the isomers are at least $13000 \mathrm{~K}$ higher in energy. $\left(\mathrm{NH}_{4} \mathrm{Cl}\right)_{5}$ has a few low-energy isomers (see Fig. 9), but there is a large gap between the group of low-energy isomers and the group of open-type higher energy isomers. $\left(\mathrm{NH}_{4} \mathrm{Cl}\right)_{6}$ has just one other low energy isomer in addition to the global minimum, and the energy difference between the two low energy isomers in $\left(\mathrm{NH}_{4} \mathrm{Cl}\right)_{6}$ is roughly the same as the difference between the two isomers of $\left(\mathrm{NH}_{4} \mathrm{Cl}\right)_{7}$ that dominate the histogram in Fig. 10(c). However, in contrast to the case of $\left(\mathrm{NH}_{4} \mathrm{Cl}\right)_{7},\left(\mathrm{NH}_{4} \mathrm{Cl}\right)_{6}$ does not have any other low-energy isomers. The melting peak in $\left(\mathrm{NH}_{4} \mathrm{Cl}\right)_{6}$, as in the other evennumber clusters, is significantly larger compared to the $T$ $=0 \mathrm{~K}$ heat capacity than in the odd-number clusters. Any decline in the heat capacity accompanying the onset of rapid isomerization between the two low-lying isomers of $\left(\mathrm{NH}_{4} \mathrm{Cl}\right)_{6}$ is masked in the $800 \mathrm{~K}$ region by the melting peak. Conversely, the melting peak in the heat capacity of

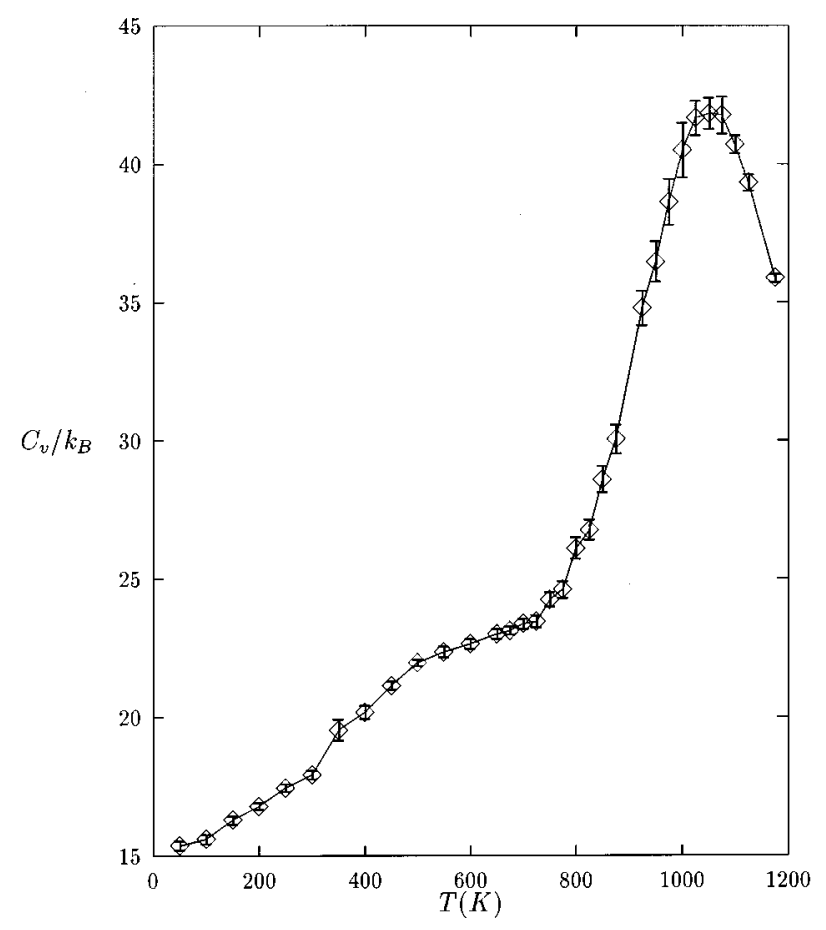

FIG. 7. The potential energy contribution to the constant-volume heat capacity for $\left(\mathrm{NH}_{4} \mathrm{Cl}\right)_{4}$ as a function of $T$. The maximum at $1100 \mathrm{~K}$ is defined to be a melting peak.

$\left(\mathrm{NH}_{4} \mathrm{Cl}\right)_{7}$ is small and does not mask the drop in the heat capacity.

The structure (Fig. 3(d)) and the heat capacity of $\left(\mathrm{NH}_{4} \mathrm{Cl}\right)_{8}$ (Fig. $7(\mathrm{~d})$ ) are similar to those of $\left(\mathrm{NH}_{4} \mathrm{Cl}\right)_{6}$ (see Figs. 3(b) and 7(b), respectively). The slope of the curve in the $500-800 \mathrm{~K}$ "shoulder" region is not as flat as in the case of $\left(\mathrm{NH}_{4} \mathrm{Cl}\right)_{6}$. Similar to $\left(\mathrm{NH}_{4} \mathrm{Cl}\right)_{6}$, there are a few lowenergy isomers with compact structures accessible at lower temperatures, and the majority of isomers are higher-energy open-type structures that become accessible in the vicinity of the melting peak.

The $\left(\mathrm{NH}_{4} \mathrm{Cl}\right)_{9}$ cluster, shown in Fig. 3(e), has more in common with the even-number clusters than it does with the odd-number clusters. The structure of $\left(\mathrm{NH}_{4} \mathrm{Cl}\right)_{9}$ is slab-like, reminiscent of rock-salt structure of $\mathrm{NaCl}$. The heat capacity curve, shown in Fig. 7(e), shows a flat region in the 400-800 $\mathrm{K}$ region and a large melting peak at about $1100 \mathrm{~K}$. An interesting feature of the $\left(\mathrm{NH}_{4} \mathrm{Cl}\right)_{9}$ cluster is the low rotational barrier of the ammonium ion in the center of the cluster. The center ammonium ion has 12 identical potential energy minima as it rotates by 360 degrees, and the barrier to rotation between each pair of minima is only $48 \mathrm{~K}$. Although we can see the center ammonium ion hopping between different minima at temperatures as low as $5 \mathrm{~K}$, the contribution of this motion to the heat capacity is small and masked by the steady rise in the heat capacity from the anharmonic vibrational motions in the rest of the cluster.

We have claimed that the main contributing factor to the appearance of a temperature region where the slope of $C_{v}$ is 


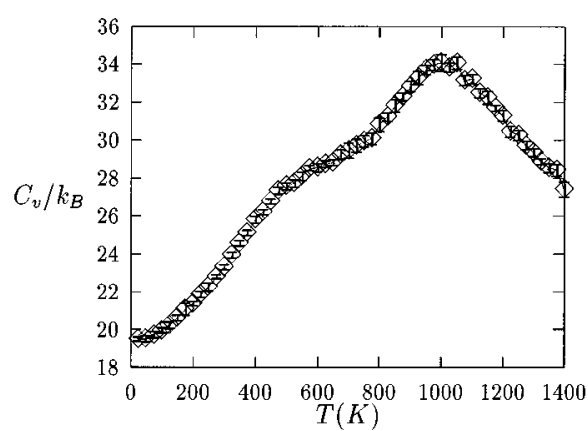

(a)

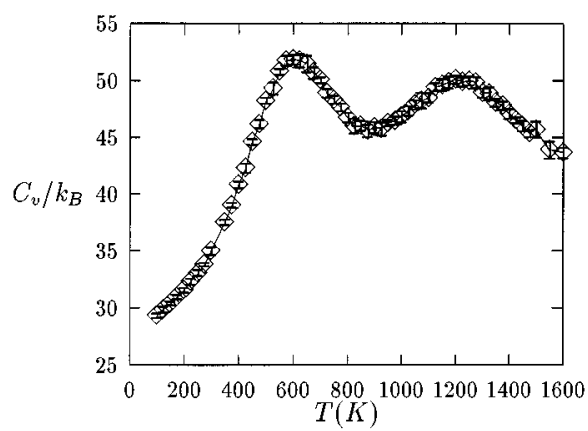

(c)

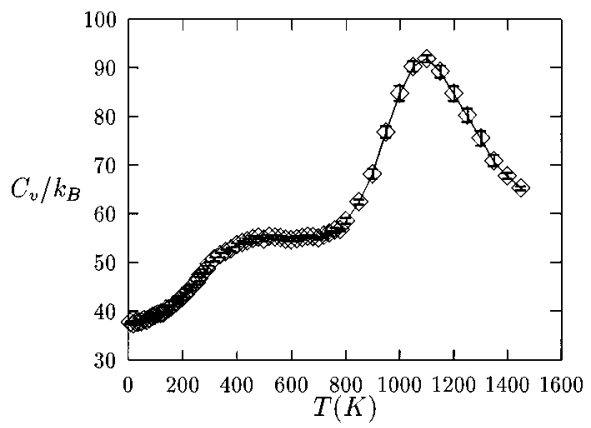

(e)

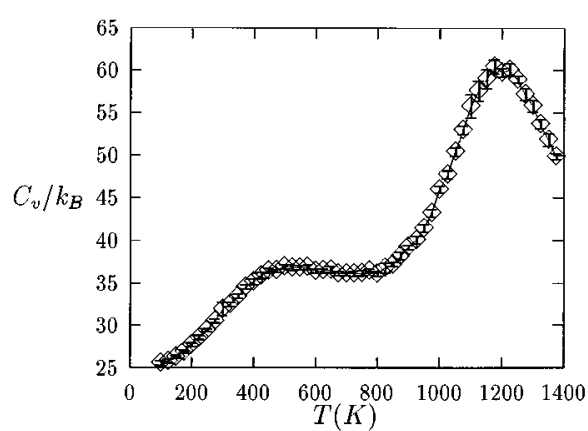

(b)

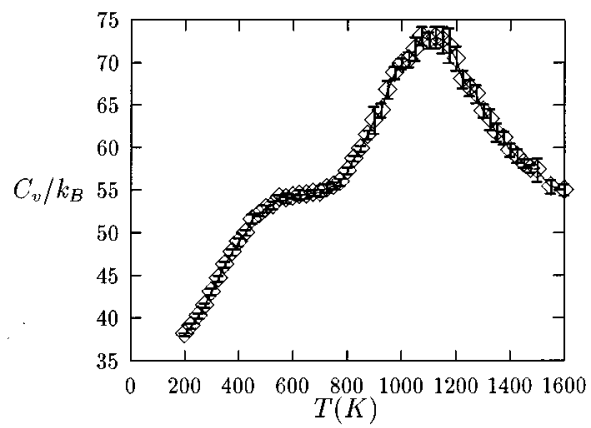

(d)

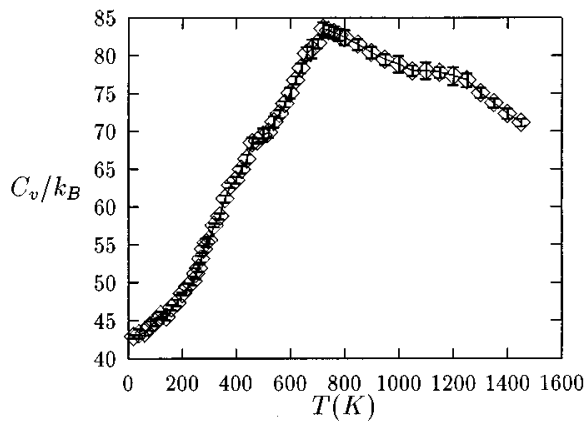

(f)

FIG. 8. The potential energy contribution to the constant-volume heat capacities for $\left(\mathrm{NH}_{4} \mathrm{Cl}\right)_{n}, n=5-10$, where (a) $n=5$, (b) $n=6$, (c) $n=7$, (d) $n=8$, (e) $n=9$, and (f) $n=10$.

small is the onset of free rotation of the ammonium ions. As a qualitative test of this claim we carry out two additional Metropolis Monte Carlo simulations in this temperature range for $\left(\mathrm{NH}_{4} \mathrm{Cl}\right)_{9}$. In the first simulation, only the rotational Monte Carlo moves are allowed, with the translational moves of the ammonium and chloride ions excluded from the simulation. In the second simulation, the translational moves of the ammonium and chloride ions are included, but the rotational moves of the ammonium ions are not allowed. It must be stressed that this calculation has been done to demonstrate qualitatively the effects of the onset of free rotation on the heat capacity. The two curves are shown in Fig. 11. The upper curve is the heat capacity resulting from the translational moves, and the lower curve includes only rotations. The upper (translational) curve increases through the entire temperature range in Fig. 11. The lower (rotational) curve rises to a maximum at about $600 \mathrm{~K}$ and then undergoes a decrease. The increase in the rotational contribution to the heat capacity coincides with the ammonium ions undertaking infrequent hops between the equivalent orientations. Above $600 \mathrm{~K}$, however, the motions of the ammonium ions are more representative of free rotation rather than hops, which is consistent with the decreasing rotational contribution to the heat capacity. We have confirmed this by examining the configurations of the $\left(\mathrm{NH}_{4} \mathrm{Cl}\right)_{9}$ cluster at several temperatures throughout the flat region of the heat capacity. The rotational barrier of a corner ammonium ion is $2908 \mathrm{~K}$, and the rotational barrier of a side ammonium ion is $1948 \mathrm{~K}$. Both of these values are consistent with the onset of free rotation at $600 \mathrm{~K}$. The sum of the rotational and translational contributions is nearly flat as in the full $C_{v}$ curve shown in Fig. 8(e).

The heat capacity for the remaining cluster in the study, $\left(\mathrm{NH}_{4} \mathrm{Cl}\right)_{10}$, is shown in Fig. 8(f) and the lowest-energy iso- 


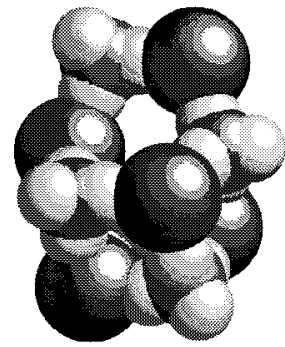

(a)

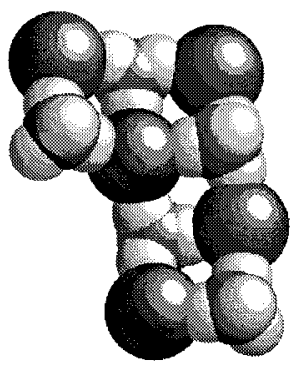

(c)

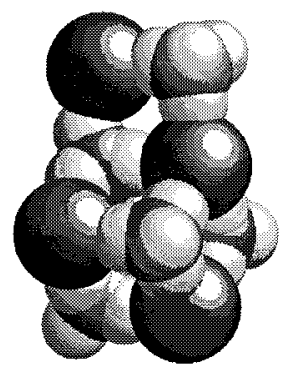

(b)

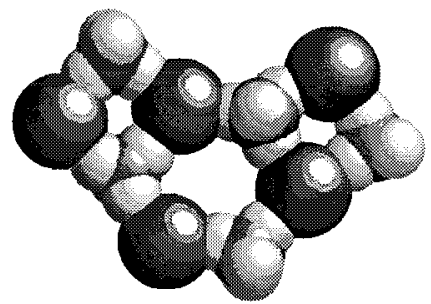

(d)
FIG. 9. (a) and (b) are examples of compact structures of $\left(\mathrm{NH}_{4} \mathrm{Cl}\right)_{5}$, with energies of $160 \mathrm{~K}$ and $773 \mathrm{~K}$ above the lowest-energy isomer, and (c) and (d) are examples of open-type structures, with energies $12850 \mathrm{~K}$ and 15079 $\mathrm{K}$ above the lowest energy isomer, respectively.

mer is shown in Fig. 3(f). The heat capacity rises steadily, reaches its maximum at $700 \mathrm{~K}$ and then slowly decreases with increasing temperature. There is a change in slope in the $1000-1200 \mathrm{~K}$ region, where clusters of size 4-9 exhibit a well-defined peak.

\section{CONCLUDING REMARKS}

In this paper we have presented a study of structures and temperature-dependent thermodynamic properties of $\left(\mathrm{NH}_{4} \mathrm{Cl}\right)_{n}$ clusters, $n=3-10$. The structures have been determined using the approach of simulated annealing, and the constant-volume heat capacities have been computed using a newly developed parallel PVM J-walking algorithm.

The lowest energy isomers of the even-number clusters form similar slab-like structures (see Figs. 3(a), 4(b), 4(d), and 4(f)). The lowest-energy isomers of the odd-number clusters (see Figs. 2(a), 4(a), 4(c), and 4(e)) generally do not show a clear similarity with either the preceding evennumber cluster or the previous odd-number cluster.

We can compare our results to the structures of $(\mathrm{NaCl})_{n}$ clusters determined by Phillips et al. ${ }^{7}$ For $(\mathrm{NaCl})_{3}$, only one isomer is found, compared to three for $\left(\mathrm{NH}_{4} \mathrm{Cl}\right)_{3}$. The structure of $(\mathrm{NaCl})_{3}$ is a planar ring, similar to the highest energy isomer of $\left(\mathrm{NH}_{4} \mathrm{Cl}\right)_{3}$, shown in Fig. 2(e). A close

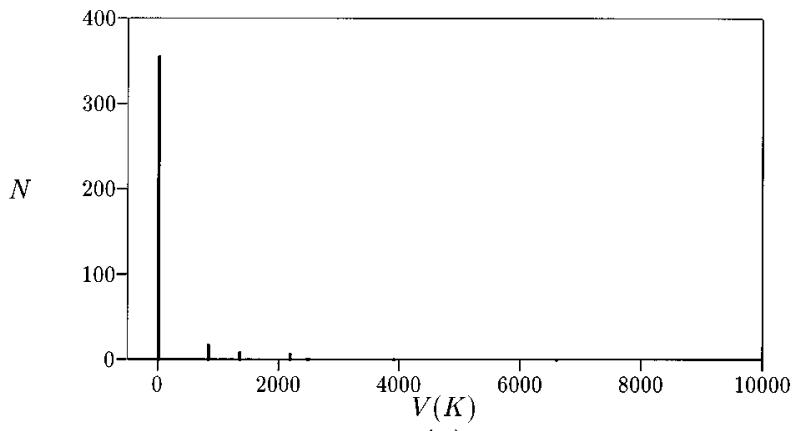

(a)

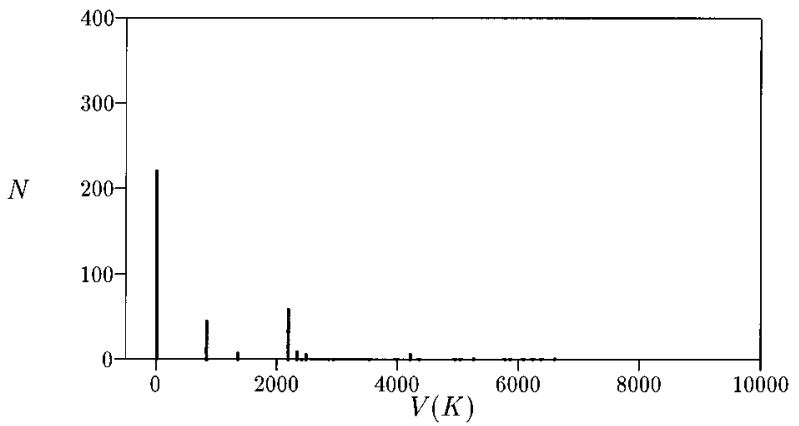

(b)

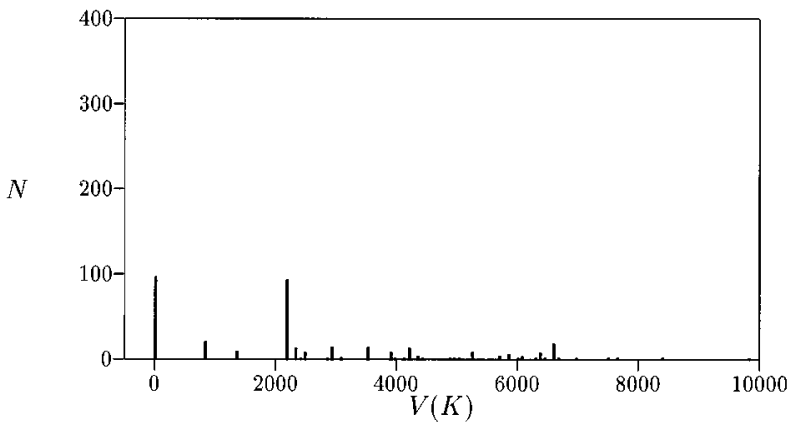

(c)

FIG. 10. Histograms of isomer distribution for $\left(\mathrm{NH}_{4} \mathrm{Cl}\right)_{7}$ at (a) $425 \mathrm{~K}$, (b) $600 \mathrm{~K}$, and (c) $800 \mathrm{~K}$. The units of potential energy $V$ in these graphs are degrees $\mathrm{K}$ above the potential energy of the lowest energy isomer. The variable $N$ is the number configurations in a Monte Carlo walk whose nearest local minimum on the potential energy surface has potential energy $V$.

examination of the $\left(\mathrm{NH}_{4} \mathrm{Cl}\right)_{3}$ isomer shown in Fig. 2(c) together with the absence of such a structure in $(\mathrm{NaCl})_{3}$ suggests that the $\left(\mathrm{NH}_{4} \mathrm{Cl}\right)_{3}$ isomer shown in Fig. 2(c) is stabilized by the attractive hydrogen-chloride interactions. The geometries of the lowest energy isomers for $(\mathrm{NaCl})_{n}, n$ $=4,7,8,10$ are similar to the geometries of the lowest energy isomers that we find for the respective $\left(\mathrm{NH}_{4} \mathrm{Cl}\right)_{n}$ clusters. For the remaining isomers, $n=5,6$, and 9 , the geometries of the second lowest isomers of $(\mathrm{NaCl})_{n}$ are similar to those of the lowest energy isomers of $\left(\mathrm{NH}_{4} \mathrm{Cl}\right)_{n}$. In the work of Diefenbach and Martin, ${ }^{33}$ lowest energy isomers are identified for several alkali halide clusters. They find that the lowest energy isomer geometries are dependent on both the constitu- 


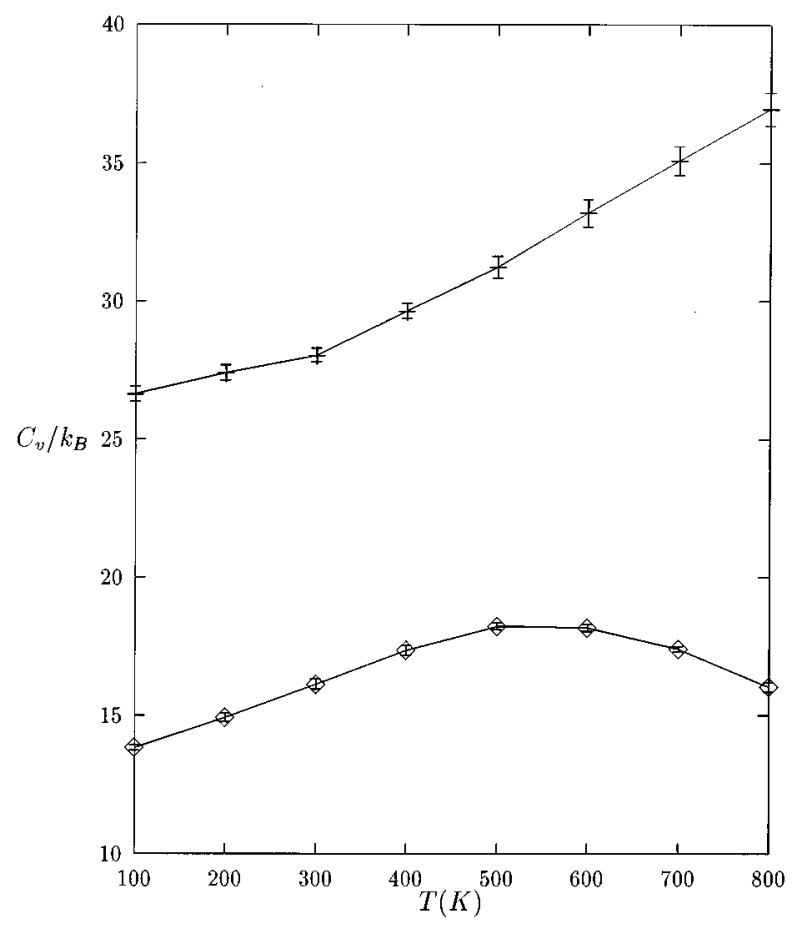

FIG. 11. Upper curve shows the constant-volume heat capacity for $\left(\mathrm{NH}_{4} \mathrm{Cl}\right)_{9}$ in the absence of rotational moves, and the lower curve is the heat capacity with the translational motion of ammonium and chloride ions excluded. The sum of the two curves changes little explaining the plateau features in Fig. 8.

ent ions and the potential model used. It is noteworthy that unlike bulk ammonium chloride crystal, for which the cesium chloride structure is thermodynamically stable, we have found no cesium chloride type structures in the clusters. The cluster size at which the cesium chloride structure begins to appear represents an interesting problem.

A major thrust of this paper is the development of the parallel J-walking algorithm with the use of parallel virtual machine (PVM) package. The ability to maintain the J-walking distributions dynamically at each required temperature makes it possible to perform an ergodic Monte Carlo simulation spanning the entire temperature range of interest. The PVM J-walking algorithm is designed to take full advantage of a multi-processor computing environment. Ergodic simulations spanning the entire temperature range of interest require the same amount of time as a single Metropolis Monte Carlo simulation, provided that a separate processor can be assigned to each process in a PVM algorithm. PVM is designed to be most efficient when the passing of information among the different processes is infrequent. In that respect, PVM is ideally suited for J-walking simulations because the most frequent jumps are made only once every 15 passes in the computing processes. The PVM J-walking algorithm does not require the storage of large external configuration files needed in the serial algorithm, and ergodic Monte Carlo simulations can be performed on significantly larger systems than possible previously.

The constant-volume heat capacities for each cluster size have been determined, revealing two important temperature regions. The first region, seen most prominently in the $\left(\mathrm{NH}_{4} \mathrm{Cl}\right)_{n}, n=4,6,8$, and 9 clusters, is the flat region in the $500-800 \mathrm{~K}$ vicinity. This flat region in the heat capacity is apparently a consequence of competing contributions from rotational motion of the ammonium ions and the anharmonic vibrational motion in the clusters. The other feature of the heat capacity curves for the majority of the clusters is the prominent maximum in the $1000-1200 \mathrm{~K}$ range that we have called a melting peak. This peak coincides with isomerization to open-type configurations. Cluster geometries at temperatures below $1000 \mathrm{~K}$ are normally dominated by compact structures belonging to potential energy wells of low lying isomers.

Future work on ammonium chloride clusters will be directed toward a quantum mechanical treatment of this system. J-walking has already been extended to quantum systems by incorporating it into the Fourier path integral Monte Carlo method. ${ }^{2}$ Realistic inclusion of all degrees of freedom in this system cannot be accomplished using classical mechanics because of the intrinsic quantum-mechanical nature of the internal vibrations of the ammonium ions. As an example of the potential importance of quantum contributions, it can be anticipated that the quantum-mechanical treatment will have a significant effect on the heat capacity curve of the $\left(\mathrm{NH}_{4} \mathrm{Cl}\right)_{3}$ cluster because the differences in zero-point energies of the isomers can be expected to rearrange the ordering and change the energy spacings between the isomers. Furthermore, significant isotope effects are known in the bulk system, implying the importance of quantum effects in the clusters. $^{34}$

\section{ACKNOWLEDGMENTS}

This work was supported in part by the National Science Foundation under Grant Nos. CHE-9411000 and CHE9203498. Acknowledgement is made to the Donors of the Petroleum Research Fund of the American Chemical Society for partial support of this work. This research was sponsored in part by the Phillips Laboratory, Air Force Material Command, USAF, through the use of the MHPCC under cooperative agreement number F29601-93-2-0001. The views and conclusions contained in this document are those of the authors and should not be interpreted as necessarily representing the official policies or endorsements, either expressed or implied, of Phillips Laboratory or the U.S. Government. We thank Jim Doll for helpful discussions and suggestions.

${ }^{1}$ D. D. Frantz, D. L. Freeman, and J. D. Doll, J. Chem. Phys. 93, 2769 (1990).

${ }^{2}$ D. D. Frantz, D. L. Freeman, and J. D. Doll, J. Chem. Phys. 97, 5713 (1992)

${ }^{3}$ D. D. Frantz, J. Chem. Phys. 102, 3747 (1995).

${ }^{4}$ T. L. Beck, and R. S. Berry, J. Chem. Phys. 88, 3910 (1988); J. Jellinek, T. L. Beck, and R. S. Berry, ibid. 84, 2783 (1986).

${ }^{5}$ R. S. Berry, J. Jellinek, and G. Natanson, Phys. Rev. A 30, 919 (1994).

${ }^{6}$ C. J. Tsai and K. D. Jordan, J. Phys. Chem. 97, 11227 (1993).

${ }^{7}$ N. G. Phillips, C. W. S. Conover, and L. A. Bloomfield, J. Phys. Chem. 94, 4980 (1991).

${ }^{8}$ H. L. Davis, D. J. Wales, and R. S. Berry, J. Chem. Phys. 92, 4308 (1990),

${ }^{9}$ D. J. Wales, J. Chem. Faraday Trans. 89, 1305 (1993). 
${ }^{10}$ R. S. Berry, T. L. Beck, H. L. Davis, and J. Jellinek, Adv. Chem. Phys. 70B, 75 (1988).

${ }^{11}$ J. P. Rose and R. S. Berry, J. Chem. Phys. 96, 517 (1992).

${ }^{12}$ R. M. Lyndon-Bell and D. J. Wales, J. Chem. Phys. 101, 1460 (1994).

${ }^{13}$ G. E. Lopez and D. L. Freeman, J. Chem. Phys. 98, 1428 (1993).

${ }^{14}$ C. Kittel, Introduction to Solid State Physics (Wiley, New York, 1971), 4th ed.

${ }^{15}$ N. Metropolis, A. Rosenbluth, M. N. Rosenbluth, A. Teller, and E. Teller, J. Chem. Phys. 21, 1087 (1953).

${ }^{16}$ G. A. Geist, A. L. Beguelin, J. J. Dongarra, W. Jiang, R. J. Manchek, and V. S. Sunderam, PVM: Parallel Virtual Machine - A Users Guide and Tutorial for Network Parallel Computing, (MIT, Cambridge, 1994).

${ }^{17}$ M. L. Klein, I. R. McDonald, and Y. Ozaki, J. Chem. Phys. 79, 5579 (1983)

${ }^{18}$ B. M. Pettitt and P. J. Rossky, J. Chem. Phys. 84, 5836 (1986).

${ }^{19}$ B. M. Pettitt and P. J. Rossky, J. Chem. Phys. 77, 1451 (1982).

${ }^{20}$ J. Lubkowski and J. Blazejowski, J. Phys. Chem. 95, 2311 (1991).

${ }^{21}$ J. W. Wilson, J. Chem. Soc., Dalton Trans. Part 1, 890 (1976).

${ }^{22}$ J. K. Lee, J. A. Barkar, and F. F. Abraham, J. Chem. Phys. 58, 3166 (1973).
${ }^{23}$ D. L. Freeman and J.D. Doll, Adv. Chem. Phys. 70B, 139 (1988).

${ }^{24}$ G. Herzberg, Molecular Spectra and Molecular Structure II. Infrared \& Raman Spectra of Polyatomic Molecules (Van Nostrand, New York, 1945), pp. 181-183.

${ }^{25}$ E. B. Harvey and N. R. McQuaker, J. Chem. Phys. 55, 4396 (1971).

${ }^{26}$ S. Chandrasekhar, Rev. Mod. Phys. 81, 1 (1943).

${ }^{27}$ C. Zhang, D. L. Freeman, and J. D. Doll, J. Chem. Phys. 91, 2489 (1989);

M. A. Strozak, G. E. Lopez, and D. L. Freeman, ibid. 97, 4445 (1992).

${ }^{28}$ A. Matro, D. L. Freeman, and J. D. Doll, J. Chem. Phys. 101, 10458 (1994).

${ }^{29}$ J. D. Doll, T. L. Beck, and D. L. Freeman, Int. J. Quantum Chem. Quantum Chem. Symp. 23, 73 (1989).

${ }^{30}$ R. L. Hildenbrandt, Comp. Chem. 1, 175 (1977)

${ }^{31}$ C. J. Cerjan and W. H. Miller, J. Chem. Phys. 75, 280 (1981); J. Simons, P. Jorgensen, H. Taylor, and J. Ozment, J. Phys. Chem. 89, 52 (1985).

${ }^{32}$ N. W. Howard and A. C. Legon, J. Chem. Phys. 88, 4694 (1988).

${ }^{33}$ J. Diefenbach and T. P. Martin, J. Chem. Phys. 83, 4585 (1985).

${ }^{34}$ N. G. Parsonage and L. A. K. Staveley, Disorder in Crystals (Clarendon, Oxford, 1978), p. 311 
The Journal of Chemical Physics is copyrighted by the American Institute of Physics (AIP). Redistribution of journal material is subject to the AIP online journal license and/or AIP copyright. For more information, see http:/ojps.aip.org/jcpo/jcpcr/jsp Copyright of Journal of Chemical Physics is the property of American Institute of Physics and its content may not be copied or emailed to multiple sites or posted to a listserv without the copyright holder's express written permission. However, users may print, download, or email articles for individual use. 
The Journal of Chemical Physics is copyrighted by the American Institute of Physics (AIP). Redistribution of journal material is subject to the AIP online journal license and/or AIP copyright. For more information, see http://ojps.aip.org/jcpo/jcper/jsp 